\title{
Time-to-passage judgments in nonconstant optical flow fields
}

\author{
MARY K. KAISER and HEIKO HECHT \\ NASA Ames Research Center, Moffett Field, California
}

The time until an approaching object will pass an observer (time to passage, or TTP) is optically specified by a global flow field even in the absence of local expansion or size cues. Kaiser and Mowafy (1993) have demonstrated that observers are in fact sensitive to this global flow information. The present studies investigate two factors that are usually ignored in work related to TTP: (1) non-constant motion functions and (2) concomitant eye rotation. Non-constant velocities violate an assumption of some TTP derivations, and eye rotations may complicate heading extraction. Such factors have practical significance, for example, in the case of a pilot accelerating an aircraft or executing a roll. In our studies, a flow field of constant-sized stars was presented monocularly on a large screen. TTP judgments had to be made on the basis of one target star. The flow field varied in its acceleration pattern and its roll component. Observers did not appear to utilize acceleration information. In particular, TTPs with decelerating motion were consistently underestimated. TTP judgments were fairly robust with respect to roll, even when roll axis and track vector were decoupled. However, substantial decoupling between heading and track vector led to a decrement in performance, in both the presence and the absence of roll.

As an observer moves through the environment, the temporal ranges of objects are specified by optical variables. Specifically, the time until an object passes the observer's eye plane (i.e., the plane perpendicular to the observer's motion vector) is approximated by the function global tau $\left(\tau_{\mathrm{g}}=\theta / \dot{\theta}\right)$, where $\theta$ is the angle between the object and the heading vector, ${ }^{1}$ and $\dot{\theta}$ the temporal derivative of this angle). Examination of the derivation of $\tau_{\mathrm{g}}$ reveals that the relative observer-object velocity is assumed to be constant in order for $\tau_{\mathrm{g}}$ to be an accurate estimate of time to passage (TTP) (Kaiser \& Mowafy, 1993; Lee, 1976; Tresilian, 1991). In addition to the mathematical assumptions underlying $\tau_{\mathrm{g}}$, pragmatic assumptions concerning observers' competencies must be made. Notably, one must assume that the observer can register both the angle (or retinal distance) between the object and the observer's track vector, and the rate of change of this angle (or distance). The difficulty of extracting these parameters may be increased if the concomitant motion resulting from head rotations is added to the visual stimulus; it has been shown that such motions affect the precision of heading estimates, particularly in the absence of efferent signals

The authors would like to thank Larry Beck and Felix Shung of Sterling Software for their excellent programming support. We also thank Mike Braunstein, William Schiff, James Tresilian, and an anonymous reviewer for their extremely helpful comments and suggestions. Heiko Hecht's work was supported by a postdoctoral fellowship from the National Research Council, This research was funded by NASA RTOP 538-04-11. Portions of this research were presented at the 35th meeting of the Psychonomic Society and the 1994 meeting of the Association for Research in Vision and Ophthalmology. Correspondence concerning this article should be addressed to M. K. Kaiser, Mail Stop 262-2, NASA Ames Research Center, Moffett Field, CA 94035-1000 (e-mail: moose@eos.arc.nasa.gov).
(Perrone \& Stone, 1994; Royden, Banks, \& Crowell, 1992; Warren \& Hannon, 1990).

Most empirical research on observers' sensitivity to tau information has focused on the situation of collision rather than passage, although Schiff and Oldak (1990) have considered both collision and passage cases, and Bootsma and Oudejans (1993) have examined performance from a variety of vantage points. In the case of direct approach, the optical variable local tau $\left(\tau_{1}\right)$ approximates time to collision (TTC). ${ }^{2}$ Local tau operates on the angular expanse of the approaching object and the rate of change of that expanse; that is, local tau operates on the local expansion of the object image. For the case of passage (i.e., wherein the object passes the observer's eye plane some distance from the heading vector), local tau information is degraded since expansion is bounded by the visual angle achieved at closest approach, and because changes in the object's angular extent reflect observer-relative rotation as well as approach. In such passage situations, global tau is the appropriate optical variable. Global tau operates on the expansion of the angle (or distance) between an object and the observer's track vector; it requires a global analysis of the flow field (to establish both the track vector and the object's position relative to it). The mathematical derivations of local and global tau are formally equivalent, but the functional utility of the two information sources assume different perceptual competencies (Kaiser \& Mowafy, 1993).

The effect of nonconstant velocity on TTP and TTC has been considered previously (Lee \& Reddish, 1981; Tresilian, 1993, 1994). Essentially, all analyses of the issue agree that tau-based strategies assume constant velocity. Thus, the utilization of a tau-based strategy in the presence of acceleration (positive or negative) will result in sys- 
tematic errors in judgment. However, surprisingly little empirical data on performance exist. ${ }^{3}$ The most noteworthy studies were performed by Lee and his colleagues, examining intercept timing behavior in birds (Lee \& Reddish, 1981) and humans (Lee, Young, Reddish, Lough, \& Clayton, 1983). The findings of these studies suggest that TTC judgments are consistent with an assumption of constant approach velocities, even when acceleration was present. However, Tresilian (1993) argues that these studies may not have been sufficiently sensitive to discriminate between timing based on a constant velocity assumption and nonconstant velocity strategies.

Similarly, there is relatively little information concerning the effect of concomitant head rotation on tau-based judgments. Unlike acceleration, the presence of a $z$-axis rotation (i.e., rolling about the line-of-sight axis) does not violate the underlying assumptions of the tau derivation. However, such a rotation adds an extraneous motion component to the optic flow, which must somehow be removed to recover accurate heading (and hence TTP) information. There is an extensive analytic literature suggesting how the parsing of rotational and translational components might be accomplished (see Perrone \& Stone, 1994, for a review), but these models largely deal with $y$-axis rotations (i.e., rotations about the vertical axis that are associated with gaze fixation). Perrone (1992) does briefly consider the likely impact of roll on his detector/template model, but only to suggest that large field roll detectors might interfere with the outputs of the other detector types (see also Simpson, 1988, for a discussion of how concomitant roll would be expected to affect perfect and imperfect filter models). There have been two empirical studies of the impact of roll on TTC judgments: Simpson (1988) reported that stimulus roll degraded TTC discriminations; Freeman, Harris, and Tyler (1994) found that TTC estimates were unaffected by the addition of $z$-axis rotations. There were, however, important differences in the two sets of studies. Freeman et al.'s stimuli consisted of an annular pattern of constant-sized dots; Simpson's stimuli were expanding forms (crosses). The rotational velocity in the Freeman et al. study was less than $6 \% \mathrm{sec}$; Simpson's stimuli rotated at $45 \% \mathrm{sec}$. Thus, as in the case of nonconstant velocity, findings concerning the impact of roll on TTC judgments are inconclusive.

All previous studies on nonconstant velocity and concomitant roll have examined TTC situations; none have considered passage events. Because the findings of these studies are inconclusive, and since local and global tau variables assume different perceptual competencies, an examination of the impact of acceleration and roll for TTP judgments is required. The studies we report investigate how observers' global TTP judgments are affected by the presence of acceleration and observer head rotation. Our stimuli were constructed so that no local tau information was available to observers. In the first experiment, we examined the effect of a constant linear acceleration/deceleration on TTP estimates. In the second experiment, we examined the effect of introducing a constant-velocity $z$ axis rotation (i.e., roll) about the observer's track vector. In the final experiment, we examined the impact on judgments when the roll axis was decoupled from the track vector.

\section{EXPERIMENT 1 Acceleration and Deceleration}

The first experiment assessed the accuracy of observers' TTP judgments under constant linear deceleration and acceleration conditions as opposed to zero acceleration (constant velocity). Since the target stimulus maintained a constant projected size, no local (i.e., withinobject) tau information was available. Likewise, binocular and accommodative cues to proximity were absent from the display. Thus, the optical information specifying TTP was limited to those contained in the global flow (see Tresilian, 1991, for a discussion of potential global variables in addition to $\tau[\theta]$ ). Owing to the inconclusive findings concerning the impact of acceleration on TTC judgments, we held no firm hypothesis concerning passage judgments in the presence of nonconstant velocity flow fields: observers might be sensitive to acceleration information and adjust their TTP estimates accordingly, or they might make TTP estimates that ignore the constant acceleration of the flow field presented to them.

\section{Method}

Observers. Eight male observers were recruited by an on-site organization and paid for their participation. Most attended local colleges and universities. They ranged in age from 20 to 44 years and had normal or corrected-to-normal vision.

Apparatus and Stimuli. The stimuli were generated on a Silicon Graphics Onyx $\mathrm{RE}^{2}$ workstation with a resolution of $1,280 \times 1,024$ pixels and a refresh rate of $60 \mathrm{~Hz}$ (noninterlaced). The animation update rate was 60 frames/sec. The display was routed via an Electrohome RGB rear-projection system to a large screen $(244 \times 183 \mathrm{~cm})$. The observer was seated $300 \mathrm{~cm}$ away from the screen in a heightadjustable chair to center his line of sight at the center of the display screen; the display subtended $44.2^{\circ}$ visual angle horizontally $X$ $33.9^{\circ}$ vertically.

The stimulus displays depicted a cloud of single-pixel white dots ("stars") that were randomly positioned in a virtual 3-D viewing volume (see Figure 1). One three-pixel red dot served as the target. Neither the target nor background dot size varied as a function of depicted distance. The depth of the volume was constructed so that the observer's eye-point did not traverse more than $50 \%$ of the volume at the point at which the target passed the eye plane. The depth of the volume was assigned the arbitrary scale of 1,000 graphical units (gu). Average eye-point translation was $60 \mathrm{gu} / \mathrm{sec}$. The track vector was always centered on the display. A total of 800 dots was used; dots that exited the viewing volume were not replaced. During pilot testing, it was determined that the thinning out of stars in the viewing volume as the observer moved forward was less distracting than the coming-into-existence of new stars, which would have been necessary to keep the number of visible stars constant throughout the trial.

Design. A three-factor, within-observer design was used. The first factor, acceleration, had five levels: two decelerating, one constant velocity, and two accelerating. In constant-velocity trials, the eye point translated at $60 \mathrm{gu} / \mathrm{sec}$; acceleration trials started with a translation rate of $25 \mathrm{gu} / \mathrm{sec}$ and accelerated by 10 or $15 \mathrm{gu} / \mathrm{sec}^{2}$; deceleration trials started with a translation rate of 100 or $130 \mathrm{gu} / \mathrm{sec}$ and decelerated by 10 or $15 \mathrm{gu} / \mathrm{sec}^{2}$. The second factor, initial target depth, had three levels. It was set at 660,490 , and $320 \mathrm{gu}$ in front of 


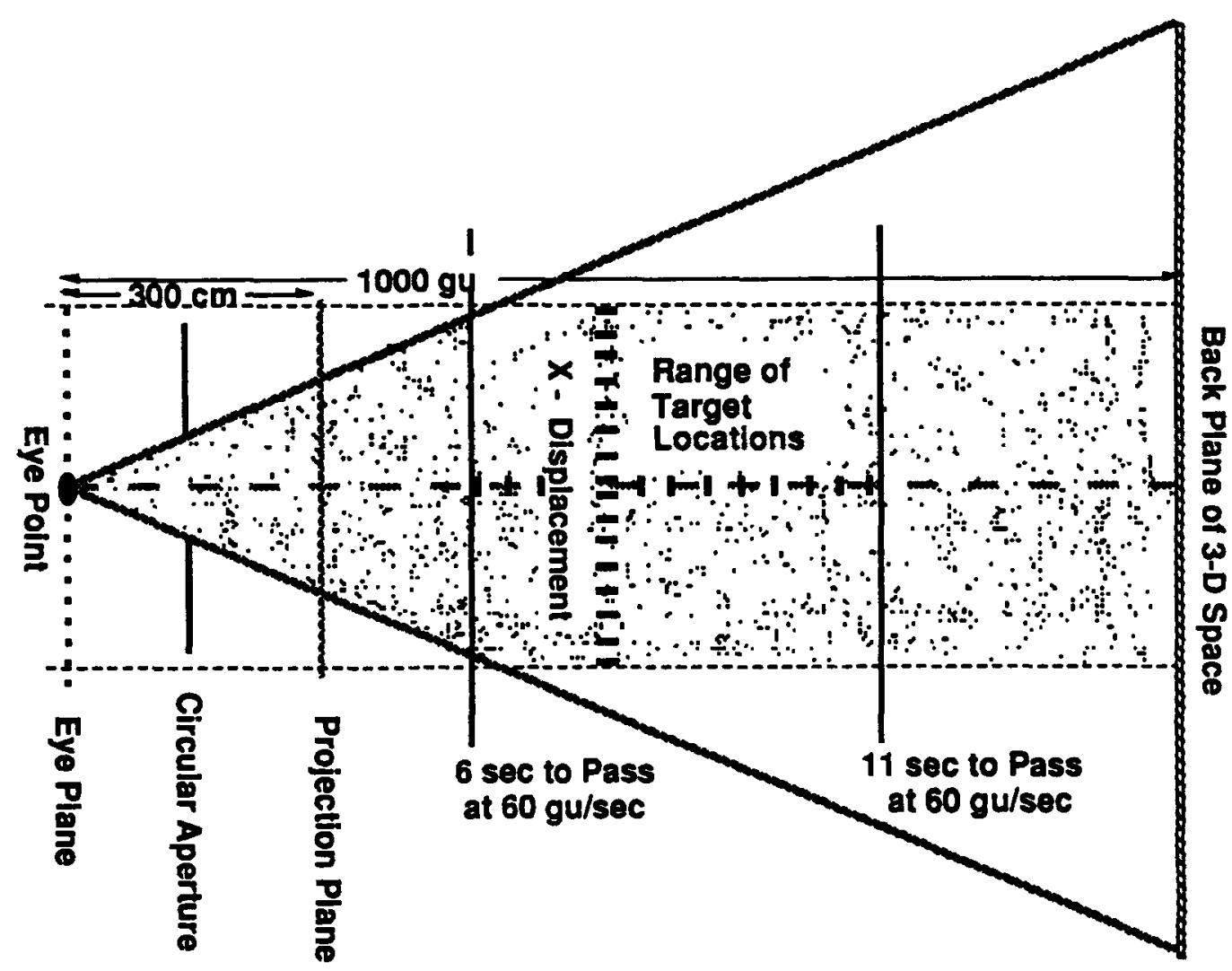

Figure 1. Schematic of the simulated viewing volume used in Experiments 1 and 2. Targets were positioned in the rear and center portion of the volume. Their positions differed in depth as well as in X-displacement. The dotted line originating at the eye point represents the track vector. The field of view (FOV) in Experiment 1 subtended $44.2^{\circ}$ horizontally $\times 33.9^{\circ}$ vertically. The circular aperture (used in Experiment 2 ) reduced the FOV to $33.7^{\circ}$ in diameter.

the eye point for acceleration and constant-velocity trials; 490, 320, and $260 \mathrm{gu}$ for decelerating stimuli. Thus, overall TTPs ranged from 3 to $11 \mathrm{sec}$. The final factor was target offset; four lateral distances between target and track vector $(15,30,45$, and 60 gu) were chosen. Varying lateral offset partially decorrelates target distance and initial angular offset (Kaiser \& Mowafy, 1993). The resulting stimulus display times and velocities are shown in Table 1.

The 60 experimental stimuli described in Table 1 were supplemented by 18 distractor trials, which were included to decorrelate the time when the target was visible on the screen (i.e., display time) with the type of motion. One distractor group consisted of decelerating trials with minimal lateral offset ( 6 or $10 \mathrm{gu}$ ), resulting in onscreen times typical of the longest experimental acceleration trials.
The other distractor group consisted of acceleration trials with extreme target offsets ( 100 or $134 \mathrm{gu}$ ); these targets remained on the screen as briefly as the shortest decelerating experimental trials. Since the target was offset from the track vector in all trials, it always exited the screen before it passed the observer. Extrapolation times ranged from 0.5 to $3.5 \mathrm{sec}$.

Procedure. The observers were seated in a dark room $3 \mathrm{~m}$ from the large screen display such that their line of sight was centered on the screen. The viewing situation was likened to a space ship pilot flying through a star field. It was explained that the target star and the white stars would not move with respect to one another (i.e., they all were fixed stars), and that the target would always go out of sight before it passed the observer. The observers were instructed to click

Table 1

Stimulus Parameters for Experiment 1

\begin{tabular}{|c|c|c|c|c|c|c|}
\hline \multirow{2}{*}{$\begin{array}{c}\text { Acceleration } \\
\left(\mathrm{gu} / \mathrm{sec}^{2}\right)\end{array}$} & \multicolumn{2}{|c|}{ Display Time in Seconds } & \multirow{2}{*}{$\begin{array}{l}\text { Initial Velocity } \\
(\mathrm{gu} / \mathrm{sec})\end{array}$} & \multicolumn{2}{|c|}{ Exit Velocity (gu/sec) } & \multirow{2}{*}{$\begin{array}{l}\text { Mean Displayed } \\
\text { Velocity Change }\end{array}$} \\
\hline & Range & $M$ & & Range & $M$ & \\
\hline \multirow[t]{2}{*}{-15} & $1.03-4.98$ & 2.34 & 100 or & $40.3-84.5$ & 64.9 & $-35.1 \%$ \\
\hline & $3.03-4.77$ & 3.84 & $130^{*}$ & $58.5-84.5$ & 72.4 & $-44.3 \%$ \\
\hline \multirow[t]{2}{*}{-10} & $1.02-3.35$ & 2.15 & 100 or & $66.5-89.8$ & 78.5 & $-21.5 \%$ \\
\hline & $2.82-4.10$ & 3.45 & $130^{*}$ & $89.0-101.8$ & 95.5 & $-26.5 \%$ \\
\hline 0 & $2.60-10.32$ & 6.46 & 60 & 60.0 & & 0 \\
\hline 10 & $3.62-8.92$ & 6.50 & 25 & $61.2-114.2$ & 90.0 & $+260 \%$ \\
\hline 15 & $3.18-7.58$ & 5.60 & 25 & $72.7-138.7$ & 109.0 & $+336 \%$ \\
\hline
\end{tabular}

*This higher initial velocity was required for the most distant deceleration targets ( $490 \mathrm{gu}$ ), to ensure that the flow field would not stop (or reverse direction) in the case of TTP overestimation. 
the left button of a three-button mouse at the point in time that the target passed their eye plane. The experimenter also informed the observers that distributing their attention would make it easier to determine the point of passage - that is, they were told that the target should not be tracked. An eye patch was worn over the observer's less preferred eye to prevent interference from inconsistent binocular depth cues. The display sequence continued until the observer clicked a mouse button to indicate the passage point. Following the observer's TTP judgment, a menu appeared, and the observer used a mouse cursor to classify the stimulus motion of the trial into one of three categories: (1) the star field slowed down, (2) the star field sped up, or (3) the star field moved at a constant rate. After the observer had categorized the stimulus motion, feedback on his TTP judgment was given (i.e., how early or late the response was in milliseconds). The next trial started automatically, after a 4-sec pause. Each observer received 15-20 practice trials with feedback (selected from the set of distractors) before the experimental trials were started.

On each trial, observers had the option of indicating that their response was in error and should be discarded (e.g., the response button was pressed accidentally). They eliminated $5.4 \%$ of the trials with this process. Trials in which observers responded more than $3 \mathrm{sec}$ after the actual TTP were also excluded; these trials accounted for $0.6 \%$ of all cases. Thus, a total of $6 \%$ of the experimental trials were omitted from the analyses.

\section{Results}

Observers' ability to compensate for nonconstant approach velocities in the TTP judgments was assessed in three ways. First, the bias (i.e., constant error) and the precision (i.e., absolute error) of TTP judgments for constant and nonconstant motion trials were compared. If observers fail to take velocity changes into account, deceleration and acceleration trials should demonstrate consistent biases: positive errors (i.e., early responses) for deceleration; negative errors (i.e., late responses) for acceleration. No particular trend is predicted for absolute error, except that longer extrapolations tend to produce more variable judgments. Second, the fits of observers' TTP judgment data to models that do and do not consider acceleration were compared. Finally, the correlation between TTP judgment error (defined as judged TTP minus actual TTP) and a variable defined as the product of acceleration rate and extrapolation time was examined.

These three convergent measures were used since it is difficult to use any single assessment to determine whether acceleration influenced judgments. This difficulty is caused by the limited sensitivity of any particular assessment; results expected from constant-velocity and accelerationbased strategies are often difficult to distinguish (Tresilian, 1993). In the present experiment, for example, the correlation between actual TTP (i.e., factoring in acceleration) and passage times based on an extrapolation of final velocity was .886. This high correlation of the models' predictions limits the sensitivity of competitive model fitting and increases the necessity for convergent measures.

The constant errors of TTP judgments were fairly similar on constant-motion and acceleration trials; observers responded significantly earlier when the motion decelerated. The average bias errors for TTP judgments are shown with striped bars in Figure 2. A repeated measures analysis of variance (ANOVA) was used to conduct planned comparisons between constant motion and the four levels of acceleration. Constant-motion TTP judgment bias was significantly different from that for decelerations of 10 $\mathrm{gu} / \mathrm{sec}^{2}[F(1,7)=5.96, p<.05]$ and $15 \mathrm{gu} / \mathrm{sec}^{2}[F(1,7)=$ $18.46, p<.005$ ], but did not differ significantly from that in the two accelerated motion conditions.

The precision of observers' judgments, as assessed by absolute error, is shown by solid bars in Figure 2. There was a significant effect for acceleration $[F(4,28)=30.92$, $p<.001]$; errors were smallest for acceleration trials, and largest for deceleration trials. This trend likely reflects the fact that greater TTP judgment variability is associated with longer extrapolation times; acceleration trials had the shortest extrapolations, constant-velocity trials had extrapolation times of intermediate length, and deceleration trials the longest. To control for this confound, the data were reanalyzed with extrapolation time as a covariant. ${ }^{4}$ This analysis of covariance revealed no significant effect for acceleration but a significant effect for extrapolation time $[F(1,398)=98.52, p<.0001]$, thus confirming that the ANOVA-based acceleration finding was an artifact of extrapolation time. Deceleration trials also had, on the average, shorter on-screen times. However, no significant correlation was noted for display time and absolute error.

Our second analysis to assess whether observers took acceleration into account involved fitting their judgment data to a constant velocity extrapolation (CVE) model strategy. According to the CVE model, observers simply base their judgments on a constant extrapolation of the target's velocity when it exits the screen. (A second constantvelocity model, based on average visible velocity, did not fit as well as the final velocity model and was subsequently abandoned.) We then compared the fit of the observers' judgments with the actual TTPs (which factors in the constant acceleration) and with hypothetical TTP values resulting from the CVE model. The observers' judgments correlated significantly more highly with the CVE model's predictions than with actual TTPs $[t(7)=2.97$, $p<.05]$; correlations were higher for the CVE model than

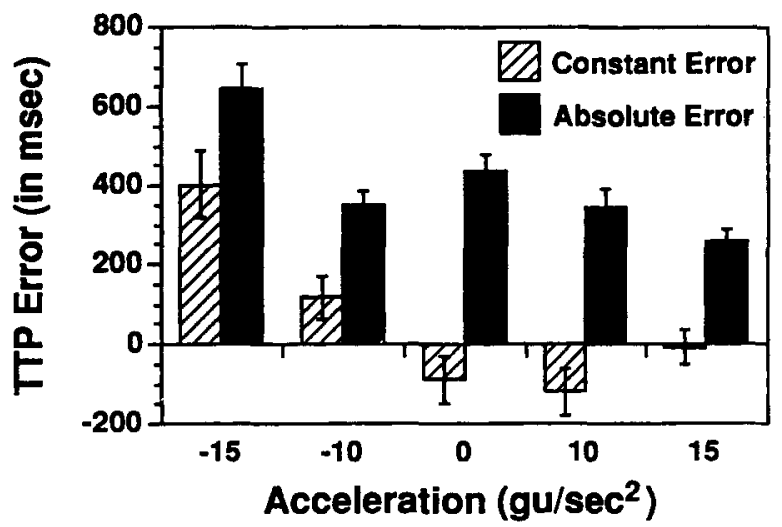

Figure 2. Average absolute and constant time-to-passage errors for the three motion conditions in Experiment 1. For the signed constant errors, positive values indicate early responses; negative values indicate late responses. Error bars depict $\pm 1 S E$. 
Table 2

Fit of Judgment Data to Actual TTP and Constant Velocity Extrapolation (CVE) Model, and Correlation of Actual TTP and TTP Based on CVE Model in Experiment 1

\begin{tabular}{lc}
\multicolumn{2}{c}{ and TTP Based on CVE Model in Experiment 1 } \\
\hline- Judged TTP with actual TTP & 0.639 \\
Judged TTP with TTP based on CVE model & 0.719 \\
Actual TTP with TTP based on CVE model & 0.886 \\
\hline
\end{tabular}

for actual TTPs for 7 of the 8 observers' data). The average correlation coefficients are shown in Table 2. A regression analysis revealed the same effect; adding an acceleration factor to a simple constant-extrapolation model did not explain a significant proportion of additional variance in observers' judgments.

Our final method to assess whether observers adjusted their TTP judgments for acceleration was to examine the correlation between a TTP error defined as (judged TTP - actual TTP) and a variable which was the product of acceleration rate and extrapolation time (e.g., $15 \mathrm{gu} / \mathrm{sec}^{2}$ * $750 \mathrm{msec}$ ). If observers did not consider acceleration, judged - actual error magnitude would increase as a multiple of these two factors. ${ }^{5}$ Separate correlations were computed for each observer. All correlation coefficients were positive, six of the eight significantly so; the average coefficient value was .382 . These positive correlations further support the CVE model.

Observers' categorization of stimulus motions was used to address the question of whether observers were even aware of the acceleration (and if such an awareness led to less biased TTP judgments). The observers reported a compelling sense of being in a moving star field, but said it was difficult to judge whether the star field speed increased or decreased. This reported uncertainty is supported by the classification data shown in Table 3 . The observers demonstrated a bias toward classifying velocities as constant; $41.2 \%$ of the accelerating stimuli and $68.4 \%$ of the decelerating stimuli were identified as such. Pearson's adjusted coefficient of contingency between stimulus motion type and observer classification was 0.316 . (A sensitivity analysis - which does not consider categorization bias-yielded $d^{\prime}$ values of $1.0,1.55$, and 0.75 for constant velocity, acceleration, and deceleration, respectively.)

Explicit awareness of the target's acceleration did not significantly decrease TTP judgment bias: $[t(5)=-0.88$ for acceleration trials - the reduced degrees of freedom resulted from the exclusion of two observers who did not

Table 3

Percent of Trials Judged as Constant, Speeding Up, or Slowing Down for Each Motion Condition

\begin{tabular}{lccc}
\hline & \multicolumn{3}{c}{ Stimulus } \\
\cline { 2 - 4 } \multicolumn{1}{c}{ Judgment } & Constant Motion & Acceleration & Deceleration \\
\hline "Constant" & 86.8 & 41.2 & 68.4 \\
"Sped up" & 5.5 & 56.1 & 11.4 \\
"Slowed down" & 7.7 & 2.7 & 20.3 \\
Total & 100 & 100 & 100 \\
\hline
\end{tabular}

misclassify any of the accelerating stimuli; $t(7)=1.25$ for deceleration trials].

\section{Discussion}

Passage events containing decelerating motions are not judged as accurately as those with constant velocity. Whereas TTP judgments for acceleration trials showed no significant bias, the short extrapolation times for these stimuli make the observation of bias unlikely; the correlation between actual TTP and TTP assuming an extrapolation of exit velocity was extremely high $(r=.98)$ for accelerating trials. For both accelerating and decelerating motions, observers' responses can be adequately modeled with a strategy based on final screen exit velocity (i.e., one that does not require the monitoring of target acceleration). It remains to be investigated whether observers will shift to different strategies if the acceleration is more extreme than the range we tested. Our results are consistent with findings obtained with a TTC paradigm (Lee et al., 1983). However, the question of whether the acceleration values used in their study were detectable (Tresilian, 1993) can also be applied to the present case. Observers may not have produced accurate TTP judgments because they did not accurately perceive the acceleration. In fact, observers correctly classified the trials as accelerating, constant, or decelerating only about half of the time, despite the fact that the resultant velocity changes in the displays were reasonably large (as shown in Table 1). In any case, the observers' TTP judgments do not reflect sensitivity to acceleration in this context.

\section{EXPERIMENT 2 Track-Coincident Roll}

The impact of $z$-axis rotation (i.e., roll about the line of sight) on TTP judgments was assessed in this experiment. In previous studies of the effect of roll on TTC discriminations and judgments (Freeman et al., 1994; Simpson, 1988), roll was centered on the object's approach axis. For simplicity and comparability, we duplicated this condition using a TTP paradigm; that is, the observer's rotational axis and track vector were coincident. However, the alignment of rotational axis with track vector resulted in an interesting property in the optic flow field; the focus of expansion (from translational motion) was likewise the point of zero rotational displacement. Thus, the 2-D image velocities traced a vortex whose center saliently specified the observer's track vector. Since the use of global tau requires extraction of the object's displacement from the track vector over time, heading-coincident roll might have two opposing effects: it could facilitate performance because it makes the track vector more salient; alternatively, roll could interfere with performance because the target continuously changes its observer-relative orientation.

\section{Method}

Observers. The same 8 observers who participated in Experiment 1 were tested in this experiment. Half of them participated in Experiment 2 first; the other half began with Experiment 1. 
Apparatus and Stimuli. The same apparatus and virtual viewing volume were used as in Experiment 1 . The constant velocity stimuli from Experiment 1 were modified by adding varying rates of $z$-axis rotation (roll). The simulated roll created a flow field such as that seen by a pilot whose airplane rotates around its longitudinal axis while flying straight ahead. That is, heading and track vectors coincided. In order to equate screen exit times for all target orientations resulting from roll, a large board with a circular aperture was put between the screen and the observer approximately $150 \mathrm{~cm}$ away from the observer. Its diameter $\left(33.7^{\circ}\right)$ was slightly less than the visual angle subtended by the vertical extent of the screen. The board was painted matte black to avoid light reflections.

Design. A three-factor (roll, initial target depth, target offset) within-observer design was used. An equal number of clockwise, counter-clockwise, and zero-roll trials were created. Velocity was constant at $60 \mathrm{gu} / \mathrm{sec}$ in all trials. Roll ranged from $-45^{\circ} / \mathrm{sec}$ to $45^{\circ} / \mathrm{sec}$ in $15^{\circ}$ steps. During pilot testing, $45^{\circ} / \mathrm{sec}$ was determined to be the largest roll that would not bear the risk of inducing motion sickness. Even small degrees of roll (e.g., $10^{\circ} / \mathrm{sec}$ ) conveyed a very compelling sense of ego rotation. Initial target depth ranged from 360 to $440 \mathrm{gu}$ in steps of $20 \mathrm{gu}$. On all trials, the eye-point translation rate was $60 \mathrm{gu} / \mathrm{sec}$. Three lateral target offset distances between target and track vector $(15,20,25 \mathrm{gu})$ were used, with offset randomly to the left or right of the track vector. The crossing of these factors resulted in a total of 105 trials. Overall TTPs ranged from 3.6 to $4.8 \mathrm{sec}$. Extrapolation times ranged from 0.4 to $1.0 \mathrm{sec}$. Target display time ranged from 2.9 to $4.4 \mathrm{sec}$ (mean $=3.5 \mathrm{sec}$ ) in all conditions.

Procedure. The same procedure was used as in Experiment 1. In addition, observers were asked to alert the experimenter if they felt nauseous. In that case, a break would be taken. Other than making TTP judgments, observers did not evaluate the stimuli. Feedback about accuracy (in milliseconds) was given following each trial.

\section{Results}

In their debriefings, the observers reported a compelling sense of motion and of rotation. As in the first experiment, observers' judgments were assessed in terms of bias (constant error) and precision (absolute error). In contrast to acceleration, it would be predicted that roll would more likely affect observers' precision (i.e., the rotational motion would add noise to observers' judgments) than add a systematic bias. Analyses of absolute error demonstrated no significant effect for roll. The introduction of moderate rates of rotation $\left(15^{\circ}\right.$ or $\left.30^{\circ} / \mathrm{sec}\right)$ about the track vector resulted in the least precise TTP judgments, but this trend was not statistically significant. Average absolute error rates for each roll magnitude are indicated by solid bars in Figure 3.

The striped bars in Figure 3 show the average constant errors plotted by roll magnitude. Here, there was a small yet significant effect for roll $[F(3,21)=3.72, p<.05]$; trend analyses indicated the quadratic effect accounted for $96 \%$ of the variance. Thus, performance was least biased in the absence of roll and at the highest roll rate $\left(45^{\circ} / \mathrm{sec}\right)$. Across all roll conditions, the trend was for observers to underestimate TTP $[M=126 \mathrm{msec} ; t(7)=5.21, p<.005]$.

\section{Discussion}

The precision of TTP judgments was fairly robust to the presence of roll. Moderate roll rates $\left(15^{\circ}\right.$ and $\left.30^{\circ} / \mathrm{sec}\right)$ produced some bias in TTP estimates and a trend toward less precision, but performance at the highest roll rate $\left(45^{\circ} / \mathrm{sec}\right)$ was virtually indistinguishable from performance in the no-roll condition. However, it should be noted that roll was always about the track vector. This coincidence of roll axis and track vector could be responsible for the robustness of TTP judgments across different degrees of roll. Since roll increased the local image velocities of the simulated stars as a function of distance to the axis of rotation (and, coincidentally, the focus of expansion), it created a spiral effect, which was likened by some observers to "water going down a drain pipe" (i.e., a vortex). This effect could have made the orientation of the track vector more salient (especially for high degrees of roll) and thus have offset any negative effects that roll might have had on extracting the target's position with respect to the track vector.

There was some evidence that a moderate rate of roll $\left(15^{\circ}-30^{\circ} / \mathrm{sec}\right)$ produced a bias in observers' judgments; TTP judgments were significantly earlier in these conditions. If this effect is reliable (it was not replicated in the next experiment), it could result from a confusion of the target's rotational and expansional motion components, a confusion that is less likely to occur when the rotational motion becomes more coherent at higher angular velocities.

To test the possibility that the coincidence of track vector and roll axis aided observer performance, these axes were decoupled in Experiment 3. Also, longer extrapolation times were added to the design to ensure that effects were not being suppressed by restricted error ranges.

\section{EXPERIMENT 3}

\section{Decoupling of Track Vector and Roll Axis}

Experiment 2 was replicated with a critical modification: The observer's roll axis and track vector were decoupled (i.e., a lateral motion component was added). The simulated flow field now corresponded to a pilot rolling his craft while deflected by a strong crosswind. That is, the aircraft was rolling about its heading axis while moving

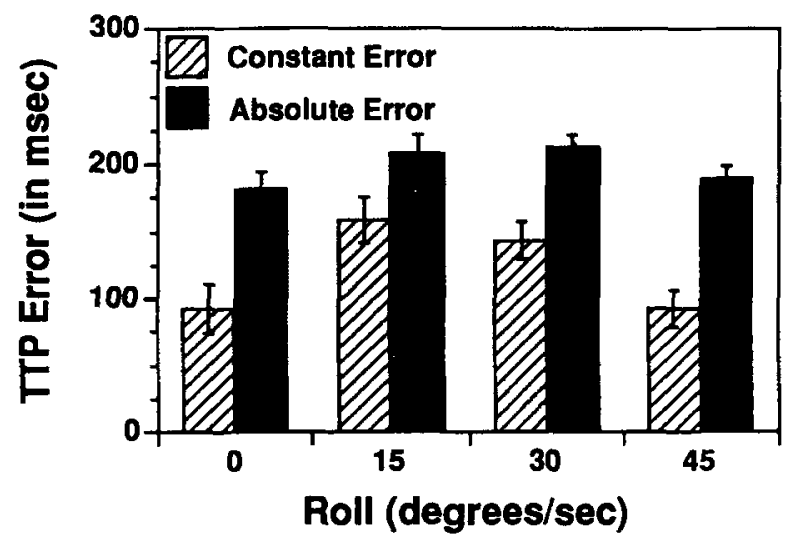

Figure 3. Average absolute and constant time-to-passage errors in Experiment 2 as a function of roll magnitude. Data have been collapsed across clockwise and counterclockwise roll within each category of roll magnitude. Error bars depict $\pm 1 S E$. 
along a track vector that deviated $0^{\circ}, 7.5^{\circ}$, or $15^{\circ}$ from the heading axis. The viewing geometry was modified to accommodate this decoupling, resulting in a deeper and wider viewing volume and longer extrapolation times.

\section{Method}

Observers. Eight male observers were paid for their participation. They ranged in age from 18 to 36 years and had normal or correctedto-normal vision. They had not participated in Experiment 1 or 2 .

Apparatus and Stimuli. The same apparatus, including the circular viewing aperture, was used as in Experiment 2. The virtual 3-D viewing volume that accommodated the cloud of single-pixel white dots ("stars") was enlarged considerably and filled with 3,000 randomly positioned stars. This was necessary because decoupling tracking and heading results in an oblique translation through the viewing volume. The volume was extended and shaped like a cone, growing wider as a function of distance from the initial eye point. The depth of the volume was enlarged to $1,500 \mathrm{gu}$; eye-point translation rate was again $60 \mathrm{gu} / \mathrm{sec}$. The targets were placed proportionally farther back in the volume, resulting in longer trials (on-screen times as well as extrapolation times). Overall TTPs ranged from 3 to $11 \mathrm{sec}$. Extrapolation times ranged from 0.5 to $3.5 \mathrm{sec}$. As before, one three-pixel red dot served as the target.

Design. A four-factor (crab angle, initial target depth, lateral target offset, and roll), within-observer design was used. The first factor, crab angle, describes the angle between the track vector (direction of simulated observer motion) and roll axis (observer heading). In the presence of crosswind, crab angle differs from $0^{\circ}$. In the previous experiments, crab angle was always $0^{\circ}$ (i.e., heading and track vector were coincident). Here, three crab angles were selected $\left(0^{\circ}\right.$, $7.5^{\circ}$, or $15^{\circ}$ ); thus, the observer was going straight through the star field or angled toward the right. Initial target depths ranged from 260 to 380 in steps of $30 \mathrm{gu}$. Lateral target offsets from the track vector were $20 \mathrm{gu}$ to the left of the observer; 20,30 , and $40 \mathrm{gu}$ to the right. Roll about the heading axis was $0^{\circ}$ or $25 \% \mathrm{sec}$. The roll rate of $25^{\circ} / \mathrm{sec}$ was chosen since it fell within the range of rates $\left(15^{\circ}-30^{\circ} / \mathrm{sec}\right)$ for which some bias was noted in Experiment 2.

Procedure. The same monocular viewing conditions and procedure were used as in Experiment 2. It was explained that the target would sometimes, but not always, move at an angle with respect to the observer. Observers were asked to factor this into their judgments about the target's intersection with the eye plane. They clicked a mouse button to indicate their TTP judgments. Feedback concerning judgment accuracy (in milliseconds) was given after each trial.

\section{Results}

The precision of TTP judgments was not affected by roll. However, a large decoupling of track and heading axes led to increased absolute error, in both the presence and absence of roll. Figure $4 \mathrm{~A}$ shows that average absolute error was significantly larger for the more extreme crab angle $\left(15^{\circ}\right)$. A repeated measures ANOVA confirmed this impression, revealing a significant effect of crab angle $[F(2,14)=19.24, p<.0001]$.

Introducing crab angle into a given set of stimuli increased extrapolation times, because the target moved obliquely to the observer's heading (as opposed to parallel to heading for a crab angle of $0^{\circ}$ ). Thus, overall increased extrapolation times might account for larger errors in the large-crab-angle condition. However, an analysis of covariance, with extrapolation time as the covariate, revealed that crab angle had a significant effect
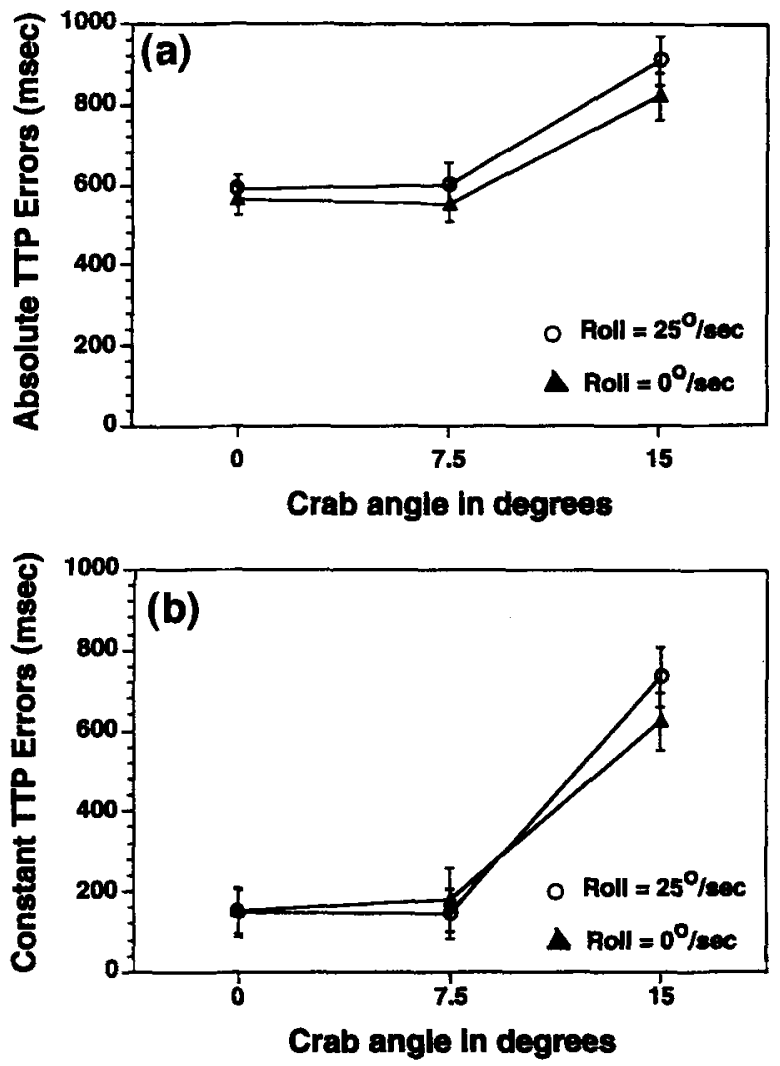

Figure 4. Average absolute (a) and constant (b) time-topassage errors in Experiment 3 as a function of roll and crab angle. Error bars depict $\pm 1 S E$.

above and beyond the contribution of extrapolation time $[F(2,860)=8.69, p<.0005]$. Extrapolation time was also a significant factor $[F(1,860)=523.87, p<.0001]$; errors increased with extrapolation time. (See note 4.)

Similarly, TTP judgments showed no bias effect of roll, but did show a significant effect of crab angle in both roll conditions. Figure 4B shows that average constant TTP errors were almost identical for the roll and no-roll conditions, but that they were significantly larger for the largecrab-angle condition. A repeated measures ANOVA confirmed this effect for crab angle $[F(2,14)=16.31, p<$ $.0002]$. As in Experiment 2, there was a trend to underestimate TTPs, but this trend was only significant for the large crab angle $[t(7)=6.79, p<.001]$.

\section{Discussion}

The more extreme $\left(15^{\circ}\right)$ decoupling of heading from track vector decreased the precision of observers' TTP judgments and introduced a significant bias toward underestimating TTP. Roll about the direction of heading does not affect performance. Observer judgments proved to be robust to potential detrimental effects of roll; the judgment bias noted for moderate roll rates in Experiment 2 was not replicated here. 


\section{GENERAL DISCUSSION}

Our findings suggest that observers do not factor acceleration into their TTP estimations. As in the TTC studies reported by Lee and his colleagues, judgments appeared to reflect a constant-velocity strategy. In our study, an extrapolation of the last seen velocity (i.e., the target's exit velocity) provided the best fit for the data. This failure to incorporate acceleration into TTP judgments is consistent with the general finding that observers are relatively insensitive to visual acceleration (e.g., Calderone \& Kaiser, 1989; Runeson, 1974) and fail to compensate for acceleration in visuomotor tracking tasks (e.g., Gottsdanker, 1952; Gottsdanker, Frick, \& Lockard, 1961). Indeed, observers were inaccurate at even identifying which trials contained acceleration, despite the significant acceleration levels in the stimuli.

TTP judgments were relatively robust to $z$-axis rotation. This was true even though the points in the visual field were not constrained to lie on a single depth plane (as were the annulus stimuli used by Freeman et al., 1994), and even for the case in which roll axis and track vector were decoupled. These findings suggest that observers can decompose the optic flow pattern in a manner sufficient to recover global tau information. Since extraction of track vector is an integral part of processing global tau information, our findings suggest that concomitant rotation of the observer's eye point has no negative effect on heading extraction. Thus, heading and temporal range extraction appear fairly robust in the presence of $z$-axis rotations, contrary to the prediction of "imperfect filter" models (Simpson, 1988).

Our finding that only large crab angles (i.e., $15^{\circ}$ ) between roll axis (and hence heading) and track vector led to larger TTP judgment errors constitutes an interesting extension of results recently reported by Navarro, Banks, and Ehrlich (1994). In their study, small crab angles (not exceeding $10^{\circ}$ ) were introduced to the trajectory of a sparse array of dots which simulated approach to the observer; no effect of crab angle was noted. Our findings suggest that significant decoupling of direction of movement (track vector) and direction of visual regard (heading) degrades TTP judgments for crab angles greater than $10^{\circ}$.

Taken together, our studies provide insight into observers' ability to estimate temporal range. Judgments reflect the tau-based assumption of constant velocity; violations to this assumption produce systematically biased judgments. As long as the roll axis and track vector are coincident, $z$-axis rotations (eye-point roll) do not disrupt global tau extraction. If they are not coincident, performance is degraded. But such degradation occurs even if no roll is present (i.e., simply when the heading and track vector are decoupled). Given that the observer's direction of motion is clearly specified by optic flow patterns as long as heading and track are coupled, these findings seem reasonable and consistent with the heading extraction literature. ${ }^{6}$ The introduction of roll about the track vector does not remove optical information for direction; in fact, $z$-axis rotation may enhance it. Rather, it is the introduction of motion components that disrupt the optical specification of motion direction (e.g., substantial crab angles) that will degrade temporal range estimation.

\section{REFERENCES}

BoOtsma, R. J., \& OUdEJANS, R. R. D. (1993). Visual information about time-to-collision between two objects. Journal of Experimental Psychology: Human Perception \& Performance, 19, 1041-1052.

CAlderone, J. B., \& Kaiser, M. K. (1989). Visual acceleration detection: Effect of sign and motion orientation. Perception \& Psychophysics, 45, 391-394.

Freeman, T. C. A., Harris, M. G., \& Tyler, P. A. (1994). Human sensitivity to temporal proximity: The role of spatial and temporal speed gradients. Perception \& Psychophysics, 55, 689-699.

GoTTSDANKER, R. M. (1952). The accuracy of prediction motion. Journal of Experimental Psychology, 43, 26-36.

GotTSDANKER, R. M., Frick, J. W., \& LoCKARd, R. B. (1961). Identifying the acceleration of visual targets. British Journal of Psychology, 52, 31-42.

JAGACINSKI, R. J., JOHNSON, W. W., \& MiLLER, R. A. (1983). Quantifying the cognitive trajectories of extrapolated movements. Journal of Experimental Psychology: Human Perception \& Performance, 9, 43-57.

KaISER, M. K., \& MowaFY, L. (1993). Optical specification of time-topassage: Observers' sensitivity to global tau. Journal of Experimental Psychology: Human Perception \& Performance, 19, 1028-1040.

LEE, D. N. (1976). A theory of visual control of braking based on information about time-to-collision. Perception, 5, 437-459.

LEE, D. N., \& REDDISH, P. E. (1981). Plummeting gannets: A paradigm of ecological optics. Nature, 293, 293-294.

Lee, D. N., Young, D. S., Reddish, P. E., Lough, S., \& Clayton, T. (1983). Visual timing in hitting an accelerating ball. Quarterly Journal of Experimental Psychology, 35A, 333-346.

MCKEE, S. P. (1981). A local mechanism for differential velocity detection. Vision Research, 21, 491-500.

Navarro, R., Banks, M. S., \& Ehrlich, S. (1994). Local and global methods for estimating time-to-contact and time-to-passage. Investigative Ophthalmology \& Visual Science, 35, 1999.

PERRONE, J. A. (1992). Model for the computation of self-motion in biological systems. Journal of the Optical Society of America A, 9, 177 194.

Perrone, J. A., \& STONE, L. S. (1994). A model of self-motion estimation within primate extrastriate visual cortex. Vision Research, 34, 2917-2938.

Rosenbaum, D. A. (1975). Perception and extrapolation of velocity and acceleration. Journal of Experimental Psychology: Human Perception \& Performance, 1, 395-403.

Royden, C. S., Banks, M. S., \& Crowell, J. A. (1992). The perception of heading during eye movements. Nature, 360, 583-585.

Runeson, S. (1974). Constant velocity-not perceived as such. Psychological Research, 37, 3-23.

SchifF, W., \& OldaK, R. (1990). Accuracy of judging time to arrival: Effects of modality, trajectory, and gender. Journal of Experimental Psychology: Human Perception \& Performance, 16, 303-316.

SCHMERLER, J. (1976). The visual perception of accelerated motion. Perception, 5, 167-185.

Simpson, W. A. (1988). Depth discrimination from optic flow. Perception, 17, 497-512.

Tresilian, J. R. (1991). Empirical and theoretical issues in the perception of time to contact. Journal of Experimental Psychology: Human Perception \& Performance, 17, 865-876.

Tresilian, J. R. (1993). Four questions of time to contact: A critical examination of research on interceptive timing. Perception, 22, 653-680.

Tresilian, J. R. (1994). Approximate information sources and perceptual variables in interceptive timing. Journal of Experimental Psychology: Human Perception \& Performance, 20, 154-173.

WARREN, W. H., \& HANNON, D. J. (1990). Eye movements and optical flow. Journal of the Optical Society of America A, 7, 160-168. 


\section{NOTES}

1. Tau derivations can be performed with either a polar coordinate system (reflecting visual angle) or a Euclidean system (reflecting retinal distance). The former utilizes the law of small angles approximation (i.e., $\tan \theta=\theta$ ); the latter approximates the retinal surface with a plane.

2. The taxonomy of tau-based variables that we utilize in this paper was set forth by Tresilian (1991).

3 . There is an extensive literature on sensitivity to visual motion acceleration (e.g., Calderone \& Kaiser, 1989; McKee, 1981; Runeson, 1974; Schmerler, 1976) and the ability to factor velocity change into motion tracking and extrapolation tasks (e.g., Gottsdanker, 1952; Gottsdanker, Frick, \& Lockard, 1961; Jagacinski, Johnson, \& Miller, 1983; Rosenbaum, 1975). However, these studies deal with motions orthogonal to the line of sight, rather than toward the observer. While the phenomena are likely related, one cannot generalize from exocentric motion analyses (and the generally poor competencies reported) to egocentric approach/passage cases.
4. Analysis of covariance treats all data points as independent, an assumption violated by our data in this and the third experiment. Thus, the effect size we report for these analyses are likely inflated (but still clearly significant); further, the null finding for acceleration in Experiment 1 is valid.

5 . That is, the larger the acceleration magnitude and the longer the period for which it is ignored, the greater the resultant error magnitude (negative for deceleration, indicative of underestimating TTP; positive for acceleration, reflecting TTP overestimation).

6. The term "heading" as used in the heading extraction literature is a misnomer. Technically, heading is the direction in which a vehicle (or body) is facing; the direction in which it is moving is its track. For most terrestrial locomotion, the two are coincident (hence the tendency for heading to be used to mean the direction of motion). We differentiate between the two terms, using track for direction of motion, and heading for direction of forward gaze.

(Manuscript received July 27, 1994; revision accepted for publication January 30,1995 .) 